\title{
Probing the tau electric dipole moment at the BEPC-II collider
}

\author{
Werner Bernreuther ${ }^{1,{ }^{*}}$ Long Chen, ${ }^{1, \dagger}$ and Otto Nachtmann ${ }^{2, *}$ \\ ${ }^{1}$ Institut für Theoretische Teilchenphysik und Kosmologie, RWTH Aachen University, \\ 52056 Aachen, Germany \\ ${ }^{2}$ Institut für Theoretische Physik, Universität Heidelberg, 69120 Heidelberg, Germany
}

(Received 7 September 2021; accepted 11 November 2021; published 3 December 2021)

\begin{abstract}
We investigate the prospects of the search for a nonzero $\tau$ EDM form factor $d_{\tau}(s)$ in $\tau$-pair production by $e^{+} e^{-}$collisions at BEPC-II collider energies. We compute the expectation values and covariances of simple and optimal $C P$-odd observables for $\tau$-pair production at $\sqrt{s}=4.18$ and $4.95 \mathrm{GeV}$ with subsequent decays of $\tau^{ \pm}$into major leptonic or semihadronic modes. For the $\tau$ decays to two pions and three charged pions, we take the full kinematic information of the hadronic system into account. Applying cuts and using realistic assumptions on the eventually attainable integrated luminosities at these energies, $\mathcal{L}(4.18)=$ $3 \times 10^{4} \mathrm{pb}^{-1}$ and $\mathcal{L}(4.95)=10^{4} \mathrm{pb}^{-1}$, respectively, we find the following. By taking into account purely semihadronic and semihadronic-leptonic $\tau^{+} \tau^{-}$decays one can achieve with optimal $C P$-odd observables the 1 s.d. sensitivities $\delta \operatorname{Re} d_{\tau}=4.5 \times 10^{-18} e \mathrm{~cm}\left(5.3 \times 10^{-18} e \mathrm{~cm}\right)$ and $\delta \operatorname{Im} d_{\tau}=2.2 \times 10^{-18} e \mathrm{~cm}$ $\left(2.7 \times 10^{-18} e \mathrm{~cm}\right)$ at $\sqrt{s}=4.18 \mathrm{GeV}(4.95 \mathrm{GeV})$.
\end{abstract}

DOI: 10.1103/PhysRevD.104.115002

\section{INTRODUCTION}

The experimental investigation of the production and decays of $\tau$ leptons at existing high-luminosity, low-energy $e^{+} e^{-}$colliders offers, among other issues, an opportunity to search for new physics beyond the standard model (SM) of particle physics. One aspect of this endeavor is the search for a nonzero $\tau$ electric dipole moment (EDM) that is a signature of $C P$ violation beyond the Kobayashi-Maskawa mechanism. As the $\tau$ lepton has a very short lifetime, the measurement of its static moments has so far not been possible, but instead, information on its nonstatic EDM form factor $^{1}$ can be extracted, for instance, from the measurement of $C P$-violating spin correlations in $\tau$-pair production in $e^{+} e^{-}$collisions. For timelike momentum transfer, the $\tau$ EDM form factor $d_{\tau}\left(q^{2}\right)$ can be a complex quantity. The best existing limits on its real and imaginary parts were obtained by the Belle I Collaboration [1] at $q^{2}=(10.58 \mathrm{GeV})^{2}$,

\footnotetext{
*breuther@physik.rwth-aachen.de

†longchen@physik.rwth-aachen.de

¥o.nachtmann@thphys.uni-heidelberg.de

${ }^{1}$ The acronym EDM is used in this paper for both the static moment and the form factor at $q^{2} \neq 0$.

Published by the American Physical Society under the terms of the Creative Commons Attribution 4.0 International license. Further distribution of this work must maintain attribution to the author(s) and the published article's title, journal citation, and DOI. Funded by SCOAP ${ }^{3}$.
}

$$
\begin{gathered}
-2.2 \times 10^{-17} e \mathrm{~cm}<\operatorname{Re} d_{\tau}\left(q^{2}\right)<4.5 \times 10^{-17} e \mathrm{~cm} \\
\text { at } 95 \% \text { C.L. }, \\
-2.5 \times 10^{-17} e \mathrm{~cm}<\operatorname{Imd} d_{\tau}\left(q^{2}\right)<0.8 \times 10^{-17} e \mathrm{~cm} \\
\text { at } 95 \% \text { C.L. }
\end{gathered}
$$

Recently. we reconsidered this topic and investigated how the sensitivity to $\operatorname{Re} d_{\tau}$ and $\operatorname{Im} d_{\tau}$ can be significantly enhanced with suitable simple and optimal $C P$ observables [2], assuming that high-statistics $\tau^{+} \tau^{-}$decay data will eventually be collected by the Belle II experiment $[3,4]$ at the KEKB collider. We analyzed also $d_{\tau}$ in some SM extensions with $C P$-violating Yukawa couplings that can generate a $\tau$ EDM much larger than the electron EDM. (Another rather recent analysis was made in [5].) We refer to [2] for an extensive list of references on $\tau$ EDM topics.

Because there is another high-luminosity, low-energy $e^{+} e^{-}$machine in operation, the Beijing BEPC-II collider, where high-statistics $\tau^{+} \tau^{-}$production and decay data are being recorded by the BESIII experiment [6], it seems appropriate and of interest to extend our analysis [2] to $e^{+} e^{-}$c.m. energies relevant for BESIII. This is what we do in this short paper. We consider the reactions

$$
e^{+}+e^{-} \rightarrow \tau^{+}+\tau^{-} \rightarrow \bar{B}+A
$$

at two $e^{+} e^{-}$c.m. energies, $\sqrt{s}=4.18 \mathrm{GeV}$ (where the $\tau$-pair production cross section is maximal) and $\sqrt{s}=4.95 \mathrm{GeV}$. At these energies the BESIII experiment has already collected a large number of $\tau$-pair events. In our 
analysis, we take the major semihadronic and leptonic $\tau$ decays, denoted by $\tau^{-} \rightarrow A$ and $\tau^{+} \rightarrow \bar{B}$, of polarized $\tau^{\mp}$ into account. Cuts are applied to the charged leptons and to the charged and neutral mesons in the final state. The SM amplitude for the production of polarized $\tau$ pairs is appended by the contribution of a $C P$-violating $\tau$ electric dipole form factor $d_{\tau}(s)$ as defined in [2] that is taken to be a complex quantity. The decays of polarized $\tau^{\mp}$ are modeled at the fully differential level with decay density matrices determined in the SM with intermediate resonances taken into account [2]. We consider suitable simple $[7,8]$ and optimal [9-11] $C P$ observables for tracing the real and imaginary part of $d_{\tau}(s)$. We compute the expectation values and covariances of these observables for the c.m. energies and reactions mentioned above and determine the statistical 1 standard deviation (s.d.) sensitivities, in particular, for the cases where (i) both $\tau$ leptons decay semihadronically and (ii) where one $\tau$ decays semihadronically and the other one leptonically.

\section{OBSERVABLES AND RESULTS}

We consider $\tau$-pair production in the reactions (2) to leading order in the SM supplemented by the $\tau$ EDM form factor. This is adequate for our purpose of computing expectation values of normalized observables. Total cross sections of the reactions (2) are used only for estimates of corresponding event numbers. At the energies of the BEPC-II collider, $Z$-boson exchange is negligible.

If one considers events where only one charged particle is measured both in $\tau^{-}$and $\tau^{+}$decay, respectively,

$$
\tau^{-} \rightarrow a\left(q_{-}\right)+X, \quad \tau^{+} \rightarrow \bar{b}\left(q_{+}\right)+X^{\prime},
$$

the following simple $C P$-odd observables can be applied $[2,7,8]$ :

$$
\begin{gathered}
\hat{T}^{i j}=\left(\hat{\mathbf{q}}_{+}-\hat{\mathbf{q}}_{-}\right)^{i} \frac{\left(\hat{\mathbf{q}}_{+} \times \hat{\mathbf{q}}_{-}\right)^{j}}{\left|\hat{\mathbf{q}}_{+} \times \hat{\mathbf{q}}_{-}\right|}+(i \leftrightarrow j), \\
T^{i j}=\left(\mathbf{q}_{+}-\mathbf{q}_{-}\right)^{i}\left(\mathbf{q}_{+} \times \mathbf{q}_{-}\right)^{j}+(i \leftrightarrow j), \\
\hat{Q}^{i j}=\left(\hat{\mathbf{q}}_{+}+\hat{\mathbf{q}}_{-}\right)^{i}\left(\hat{\mathbf{q}}_{+}-\hat{\mathbf{q}}_{-}\right)^{j}+(i \leftrightarrow j), \\
Q^{i j}=\left(\mathbf{q}_{+}+\mathbf{q}_{-}\right)^{i}\left(\mathbf{q}_{+}-\mathbf{q}_{-}\right)^{j}-\frac{1}{3} \delta^{i j}\left(\mathbf{q}_{+}^{2}-\mathbf{q}_{-}^{2}\right)+(i \leftrightarrow j) .
\end{gathered}
$$

The charged-particle three-momenta $\mathbf{q}_{\mp}$ in (4)-(7) are defined in the $e^{+} e^{-}$c.m. frame, $\hat{\mathbf{q}}_{ \pm}=\mathbf{q}_{ \pm} /\left|\mathbf{q}_{ \pm}\right|$, and $i, j \in$ $\{1,2,3\}$ denote the Cartesian vector indices. The observables (4), (5), and (6), (7) are sensitive to $\operatorname{Re} d_{\tau}(s)$ and $\operatorname{Im} d_{\tau}(s)$, respectively.

For $e^{+} e^{-}$c.m. energies considered in this paper (i.e., $\quad \sqrt{s}<5 \mathrm{GeV}$ ), and using $\hat{d}_{\tau}(s)=d_{\tau}(s) \sqrt{s} / e$, $e=\sqrt{4 \pi \alpha_{\mathrm{em}}}$, the expectation values of the observables (4)-(7) are of the form

$$
\left\langle T^{i j}\right\rangle_{a b}=c_{a b}(s) \operatorname{Re} \hat{d}_{\tau}(s) s^{i j}, \quad\left\langle\hat{T}^{i j}\right\rangle_{a b}=\tilde{c}_{a b}(s) \operatorname{Re} \hat{d}_{\tau}(s) s^{i j},
$$

$$
\left\langle Q^{i j}\right\rangle_{a b}=\kappa_{a b}(s) \operatorname{Im} \hat{d}_{\tau}(s) s^{i j}, \quad\left\langle\hat{Q}^{i j}\right\rangle_{a b}=\tilde{\kappa}_{a b}(s) \operatorname{Im} \hat{d}_{\tau}(s) s^{i j}
$$

Here, the labels $a, b$ denote charged particles from $\tau^{\mp}$ decay, cf. Eq. (3).

We evaluate (8) and (9) for the decay modes $\tau^{-} \rightarrow$ $\pi^{-}\left(q_{-}\right) \nu_{\tau}, \rho^{-}\left(q_{-}\right) \nu_{\tau}, \ell^{-}\left(q_{-}\right) \bar{\nu}_{\ell} \nu_{\tau},(\ell=e, \mu)$ and the corresponding charge-conjugate decay modes. The expectation values in (8) and (9) are defined by

$$
\begin{aligned}
\left\langle T^{i j}\right\rangle_{a b} & \equiv \frac{1}{2}\left\{\left\langle T^{i j}\right\rangle_{a \bar{b}}+\left\langle T^{i j}\right\rangle_{b \bar{a}}\right\} \\
& =\frac{1}{2}\left\{\frac{\int d \sigma_{a \bar{b}} T^{i j}}{\int d \sigma_{a \bar{b}}}+\frac{\int d \sigma_{b \bar{a}} T^{i j}}{\int d \sigma_{b \bar{a}}}\right\},
\end{aligned}
$$

and likewise for the other observables. The form (8), (9) of the expectation values holds when the phase-space cuts are $C P$ symmetric and rotational invariant. With the $e^{+}$beam direction $\hat{\mathbf{p}}$ in the $e^{+} e^{-}$c.m. frame defining the $z$ axis of the coordinate system, the symmetric traceless tensor $s^{i j}$ is given by

$$
\left(s^{i j}\right)=\frac{1}{2}\left(\hat{p}^{i} \hat{p}^{j}-\frac{1}{3} \delta^{i j}\right)=\operatorname{diag}\left(-\frac{1}{6},-\frac{1}{6}, \frac{1}{3}\right) .
$$

We calculate the expectation values of the simple $C P$ observables - and also of the optimal observables belowand the resulting statistical sensitivities to $\operatorname{Re} d_{\tau}(s)$ and $\operatorname{Im} d_{\tau}(s)$ at the $e^{+} e^{-}$c.m. energies $\sqrt{s}=4.18 \mathrm{GeV}$ and $4.95 \mathrm{GeV}$. The following cuts are applied in the $e^{+} e^{-}$c.m. frame to the transverse momenta and polar angles of the pions and charged leptons from $\tau^{\mp}$ decay $^{2}$ :

$$
p_{T}>0.04 \mathrm{GeV}, \quad|\cos \theta|<0.93
$$

In the case of the above simple observables, we use the reconstructed $\rho$ meson from of $\tau \rightarrow \rho \nu_{\tau} \rightarrow 2 \pi \nu_{\tau}$ as $\tau$-spin analyzer and apply the cuts (12) to this particle. However, the cross sections associated with this mode are calculated by applying the cuts (12) to both the charged and neutral pion from $\rho$ decay. At the c.m. energies 4.18 and $4.95 \mathrm{GeV}$, we assume the following integrated luminosities:

$$
\mathcal{L}(4.18)=3 \times 10^{4} \mathrm{pb}^{-1}, \quad \mathcal{L}(4.95)=10^{4} \mathrm{pb}^{-1} .
$$

\footnotetext{
${ }^{2}$ F. Nerling, private communication.
} 
Because the diagonal elements of the above traceless symmetric tensor observables in (8), (9) are not independent of each other, only their 3,3 components are considered that have the largest expectation values.

$\mathrm{SM}$-generated $C P$-violating effects in the flavordiagonal reaction $e^{+} e^{-} \rightarrow \tau^{+} \tau^{-}$are extremely tiny. (For a short recent discussion, see, e.g., [2].) For the $C P$ symmetric cuts (12), the SM expectation values of the above $C P$-odd observables $T^{i j}$ and $\hat{T}^{i j}$, which are also odd under naive "time reversal" $\left(T_{N}\right)$ invariance, are therefore numerically extremely small in the diagonal decay channels $a \bar{a}$. For nondiagonal decay channels, these expectation values can receive also contributions from the absorptive parts of higher-order terms in the SM amplitude, but they can be neglected for our purposes, too. The SM expectation values of $Q^{i j}$ and $\hat{Q}^{i j}$ in the diagonal decay channels $a \bar{a}$ are also negligibly small. However, for the nondiagonal decay channels, the SM expectation values of the $Q$ tensors become as large as a few $\times 10^{-2}$ in magnitude in the presence of cuts (12). Therefore, one should consider in addition to (6), (7) the observables [2]

$$
Q^{\prime i j}=Q^{i j}-\left\langle Q^{i j}\right\rangle_{0}, \quad \hat{Q}^{\prime i j}=\hat{Q}^{i j}-\left\langle\hat{Q}^{i j}\right\rangle_{0} .
$$

The label 0 indicates that the expectation value is computed in the SM. The SM expectation values of the $Q$ observables in an off-diagonal channel and the respective chargeconjugate channel are opposite in sign for $C P$-symmetric cuts,

$$
\left\langle Q^{i j}\right\rangle_{0, a \bar{b}}=-\left\langle Q^{i j}\right\rangle_{0, b \bar{a}},
$$

and likewise for $\hat{Q}^{i j}$. Thus, we have, in view of the definition (10),

$$
\left\langle Q^{\prime i j}\right\rangle_{a b}=\left\langle Q^{i j}\right\rangle_{a b}
$$

for all decay channels and likewise for $\hat{Q}^{i j}$. Thus, the values of the coefficients $\kappa_{a b}$ and $\tilde{\kappa}_{a b}$ in (9) that we compute for the tensors $Q$ hold also for the tensors $Q^{\prime}$. That is, the expectation values (10) of $Q^{i j}$ signaling a possible $\tau$ EDM are independent of $\left\langle Q^{i j}\right\rangle_{0}$. But the sensitivity to the $\tau$ EDM attainable with the measurement of this observable must be calculated from the covariance of $Q^{\prime i j}$, cf. [2]. Furthermore we note that the covariance matrix of the $T$ and $Q^{\prime}$ tensors is diagonal because the $T$ tensors are $T_{N}$ odd while the $Q^{\prime}$ tensors are $T_{N}$ even.

As to the sensitivity to the $\tau \mathrm{EDM}$, let us use as an example the measurements of $T_{33}$ and $Q_{33}^{\prime}$ in the decay channels $a \bar{b}$ and $b \bar{a}$. The resulting ideal 1 s.d. statistical errors of the dimensionful EDM couplings $\operatorname{Re} d_{\tau}$ and $\operatorname{Im} d_{\tau}$ are given by

$$
\begin{aligned}
\delta \operatorname{Re} d_{\tau}(s) & =\frac{e}{\sqrt{s}} \frac{1}{\sqrt{N_{a b}}} \frac{3\left[\left\langle T_{33}^{2}\right\rangle_{a b}\right]^{1 / 2}}{\left|c_{a b}\right|}, \\
\delta \operatorname{Im} d_{\tau}(s) & =\frac{e}{\sqrt{s}} \frac{1}{\sqrt{N_{a b}}} \frac{3\left[\left\langle Q_{33}^{\prime 2}\right\rangle_{a b}\right]^{1 / 2}}{\left|\kappa_{a b}\right|} .
\end{aligned}
$$

The event numbers of the various channels are determined by $N_{a a}=\mathcal{L} \sigma_{a a}$ and $N_{a b}=\mathcal{L} \sigma_{a b}$ for $a \neq b$, where $\sigma_{a b}=$ $\left(\sigma_{a \bar{b}}+\sigma_{b \bar{a}}\right)$ denote the respective cross sections. In the case of leptonic $\tau$ decays, we sum over $\ell=e, \mu$. The cross sections that we use for estimating the event numbers are given below in Table III.

Our results for the expectation values and square roots of the variances of the simple $C P$ observables are listed in Tables I and II for several one-prong decays of $\tau^{\mp}$. In addition, the 1 s.d. sensitivities to the real and imaginary parts of the $\tau$ EDM form factor are given that result from these numbers and from the assumed integrated luminosities (13). Our results show that at the energies of the BEPC-II collider the dimensionful observables $T_{33}$ and $Q_{33}^{\prime}$

TABLE I. Expectation values and square roots of the variances of the observables $T^{i j}$ and $\hat{T}^{i j}$ defined in (4), (5) and resulting sensitivities to $\operatorname{Re} d_{\tau}$ assuming the luminosities (13). For each channel, the first row corresponds to the $e^{+} e^{-}$c.m. energy $\sqrt{s}=4.18 \mathrm{GeV}$ and the second one to $4.95 \mathrm{GeV}$.

\begin{tabular}{cccccccc}
\hline \hline$\tau^{-} \rightarrow$ & $\tau^{+} \rightarrow$ & $c_{a b}\left(\mathrm{GeV}^{3}\right)$ & $\sqrt{\left\langle T_{33}^{2}\right\rangle_{a b}}\left(\mathrm{GeV}^{3}\right)$ & $\delta \operatorname{Re} d_{\tau}\left(\times 10^{-17} e \mathrm{~cm}\right)$ & $\tilde{c}_{a b}$ & $\sqrt{\left\langle\hat{T}_{33}^{2}\right\rangle_{a b}}$ & $\delta \operatorname{Re} d_{\tau}\left(\times 10^{-17} e \mathrm{~cm}\right)$ \\
\hline$\pi^{-} \nu$ & $\pi^{+} \bar{\nu}$ & 0.257 & 1.038 & 5.50 & 0.182 & 0.894 & 6.70 \\
& & 0.616 & 1.835 & 6.37 & 0.293 & 0.935 & 6.82 \\
$\rho^{-} \nu$ & $\rho^{+} \bar{\nu}$ & 0.041 & 0.770 & 11.77 & 0.046 & 0.890 & 12.13 \\
& & 0.098 & 1.497 & 15.08 & 0.060 & 0.985 & 16.21 \\
$\pi^{-} \nu$ & $\rho^{+} \bar{\nu}$ & 0.103 & 0.890 & 5.65 & 0.093 & 0.875 & 6.15 \\
& & 0.247 & 1.656 & 6.90 & 0.133 & 0.939 & 7.27 \\
$\ell^{-} \nu \bar{\nu}$ & $\ell^{\prime+} \bar{\nu} \nu$ & 0.021 & 0.436 & 8.79 & 0.020 & 0.829 & 17.56 \\
& & 0.050 & 0.769 & 10.23 & 0.033 & 0.878 & 17.69 \\
$\ell^{-} \nu \bar{\nu}$ & $\pi^{+} \bar{\nu}$ & -0.073 & 0.627 & 4.62 & -0.061 & 0.796 & 7.02 \\
& & -0.176 & 1.108 & 5.32 & -0.098 & 0.849 & 7.32 \\
$\ell^{-} \nu \bar{\nu}$ & $\rho^{+} \bar{\nu}$ & -0.029 & 0.568 & 7.14 & -0.031 & 0.834 & 9.80 \\
& & -0.071 & 1.061 & 8.57 & -0.044 & 0.906 & 11.81 \\
\hline \hline
\end{tabular}


TABLE II. Expectation values of the observables $Q^{\prime i j}$ and $\hat{Q}^{\prime i j}$ defined in (14) [using (16) and (9)], square roots of their variances, and resulting sensitivities to $\operatorname{Im} d_{\tau}$ assuming the luminosities (13). For each channel, the first row corresponds to the $e^{+} e^{-}$c.m. energy $\sqrt{s}=4.18 \mathrm{GeV}$ and the second one to $4.95 \mathrm{GeV}$.

\begin{tabular}{lccccccc}
\hline \hline$\tau^{-} \rightarrow$ & $\tau^{+} \rightarrow$ & $\kappa_{a b}\left(\mathrm{GeV}^{3}\right)$ & $\sqrt{\left\langle Q_{33}^{\prime 2}\right\rangle_{a b}}\left(\mathrm{GeV}^{3}\right)$ & $\delta \operatorname{Im} d_{\tau}\left(\times 10^{-17} e \mathrm{~cm}\right)$ & $\tilde{\kappa}_{a b}$ & $\sqrt{\left\langle\hat{Q}_{33}^{\prime 2}\right\rangle_{a b}} \delta \operatorname{Im} d_{\tau}\left(\times 10^{-17} e \mathrm{~cm}\right)$ \\
\hline$\pi^{-} \nu$ & $\pi^{+} \bar{\nu}$ & -0.450 & 0.914 & 2.77 & -0.224 & 0.703 & 4.28 \\
& & -1.034 & 1.346 & 2.78 & -0.384 & 0.675 & 3.76 \\
$\rho^{-} \nu$ & $\rho^{+} \bar{\nu}$ & -0.197 & 0.807 & 2.57 & -0.135 & 0.710 & 3.30 \\
& & -0.474 & 1.295 & 2.70 & -0.205 & 0.662 & 3.19 \\
$\pi^{-} \nu$ & $\rho^{+} \bar{\nu}$ & -0.325 & 0.871 & 1.75 & -0.179 & 0.709 & 2.59 \\
& & -0.759 & 1.343 & 1.82 & -0.295 & 0.679 & 2.37 \\
$\ell^{-} \nu \bar{\nu}$ & $\ell^{\prime+} \bar{\nu} \nu$ & 0.121 & 0.579 & 2.02 & 0.074 & 0.723 & 4.14 \\
& & 0.278 & 0.880 & 2.11 & 0.127 & 0.709 & 3.71 \\
$\ell^{-} \nu \bar{\nu}$ & $\pi^{+} \bar{\nu}$ & -0.168 & 0.817 & 2.62 & -0.075 & 0.733 & 5.26 \\
& & -0.385 & 1.260 & 2.76 & -0.129 & 0.725 & 4.75 \\
$\ell^{-} \nu \bar{\nu}$ & $\rho^{+} \bar{\nu}$ & -0.038 & 0.730 & 7.00 & -0.030 & 0.725 & 8.81 \\
& & -0.089 & 1.190 & 7.67 & -0.036 & 0.706 & 11.25 \\
\hline \hline
\end{tabular}

are more sensitive to the real and imaginary parts of the $\tau$ EDM than their dimensionless counterparts.

The simple $C P$ observables can be straightforwardly determined by experiment. They require the measurement of the momenta of $e, \mu$, of charged pions and also of neutral pions in order to reconstruct the momenta of $\rho^{\mp}$ in the decay $\rho^{ \pm} \rightarrow \pi^{ \pm} \pi^{0}$ in the $e^{+} e^{-}$c.m. frame, i.e., the laboratory frame of the BEPC-II collider.

A higher sensitivity to the $\tau$ EDM can be obtained with optimal observables. In addition to the one-prong decays $\tau \rightarrow \pi(q) \nu_{\tau}$ and $\tau \rightarrow \ell(q) \nu_{\ell} \nu_{\tau}$, we take now also the decays $\tau^{-} \rightarrow \pi^{-}\left(q_{1}\right) \pi^{0}\left(q_{2}\right) \nu_{\tau}$ and $\tau^{-} \rightarrow \pi^{-}\left(q_{1}\right) \pi^{-}\left(q_{2}\right) \pi^{+}\left(q_{3}\right) \nu_{\tau}$ and the corresponding charge-conjugate decay modes of $\tau^{+}$at the fully differential level into account. In the following, the labels $a, b$ denote a pair of these decays of $\tau^{\mp}$ into one, two, or three measured particles. The optimal observables for tracing $\operatorname{Re} d_{\tau}\left(\operatorname{Im} d_{\tau}\right)$ can be constructed in straightforward fashion using the term $\chi_{C P}^{R}\left(\chi_{C P}^{I}\right)$ of the $e^{+} e^{-} \rightarrow \tau^{+} \tau^{-}$production density matrix element that is proportional to $\operatorname{Re} d_{\tau}\left(\operatorname{Im} d_{\tau}\right)$ and the respective $\tau^{-} \rightarrow a+X$ and $\tau^{+} \rightarrow \bar{b}+X^{\prime}$ decay density matrices $\mathcal{D}^{a}$ and $\mathcal{D}^{\bar{b}}$. The decay density matrices involve the momenta of the particles $a$ and $\bar{b}$ in the respective $\tau^{\mp}$ rest frame. One gets

$\mathcal{O}_{R}^{a \bar{b}}=\frac{\operatorname{Tr}\left[\chi_{C P}^{R} \mathcal{D}^{a} \mathcal{D}^{\bar{b}}\right]}{\operatorname{Tr}\left[\chi_{\mathrm{SM}} \mathcal{D}^{a} \mathcal{D}^{\bar{b}}\right]}, \quad \mathcal{O}_{I}^{a \bar{b}}=\frac{\operatorname{Tr}\left[\chi_{C P}^{I} \mathcal{D}^{a} \mathcal{D}^{\bar{b}}\right]}{\operatorname{Tr}\left[\chi_{\mathrm{SM}} \mathcal{D}^{a} \mathcal{D}^{\bar{b}}\right]}$,

where the trace is taken with respect to the spin indices of $\tau^{-}$and $\tau^{+}$. The normalization involves the SM part $\chi_{\mathrm{SM}}$ of the $\tau^{+} \tau^{-}$production density matrix. Both observables are $C P$ odd. An extensive discussion of the properties of $\mathcal{O}_{R}^{a \bar{b}}$ and $\mathcal{O}_{I}^{a \bar{b}}$ and a detailed exposition and the explicit forms of the production and decay density matrices are given in [2]. We define, in analogy to (10),

$$
\left\langle\mathcal{O}_{R, I}^{a b}\right\rangle \equiv \frac{1}{2}\left[\left\langle\mathcal{O}_{R, I}^{a \bar{b}}\right\rangle+\left\langle\mathcal{O}_{R, I}^{b \bar{a}}\right\rangle\right]
$$

First, we analyze for the various decay channels and the phase-space cuts (12) whether or not the optimal $C P$-odd observables (18) may have a nonzero expectation value in the SM, which can be the case for $a \neq b$ (cf. [2]) and whether the covariance matrices have sizable nondiagonal entries. We find that the SM expectation values of $\mathcal{O}_{R}^{a \bar{b}}$ for all decay channels and those of $\mathcal{O}_{I}^{a \bar{a}}$ for the diagonal channels are very small and can be neglected for our purposes. For some nondiagonal decay channels, the SM expectation values of $\mathcal{O}_{I}^{a \bar{b}}$ can be as large as a few $\times 10^{-2}$ in magnitude. Therefore, we define [2]

$$
\mathcal{O}_{I}^{\prime a \bar{b}}=\mathcal{O}_{I}^{a \bar{b}}-\left\langle\mathcal{O}_{I}^{a \bar{b}}\right\rangle_{0}
$$

Because $\left\langle\mathcal{O}_{I}^{a \bar{b}}\right\rangle_{0}=-\left\langle\mathcal{O}_{I}^{b \bar{a}}\right\rangle_{0}$ for $C P$-symmetric cuts we have, in view of the definition (19),

$$
\left\langle\mathcal{O}_{I}^{a b}\right\rangle=\left\langle\mathcal{O}_{I}^{a b}\right\rangle .
$$

Moreover, we obtain that $\left|\left\langle\mathcal{O}_{R}^{a \bar{b}} \mathcal{O}_{I}^{\prime a \bar{b}}\right\rangle_{0}\right|<\mathrm{a}$ few $\times 10^{-4}$ with numerical uncertainties below $10^{-3}$. That is, the covariance matrix of the above optimal observables is diagonal to good approximation. Thus, within the precision of our numerical analysis, we have [cf. Eqs. (89)-(93) of [2]]

$$
\begin{aligned}
\left\langle\mathcal{O}_{R}^{a b}\right\rangle & =w_{a \bar{b}}(s) \operatorname{Re} \hat{d}_{\tau}(s) \\
\left\langle\mathcal{O}_{I}^{a b}\right\rangle & =\left\langle\mathcal{O}_{I}^{\prime a b}\right\rangle=\omega_{a \bar{b}}(s) \operatorname{Im} \hat{d}_{\tau}(s) .
\end{aligned}
$$

Using the c.m. energies $\sqrt{s}=4.18 \mathrm{GeV}$ and $4.95 \mathrm{GeV}$ and the cuts (12), we obtain the results given in Table III for the expectation values defined in Eq. (22) of the optimal 
TABLE III. Sensitivities to $\operatorname{Re} d_{\tau}$ and $\operatorname{Im} d_{\tau}$ from the expectation values (22) of the optimal observables $\mathcal{O}_{R}^{a \bar{b}}$ and $\mathcal{O}_{I}^{\prime a \bar{b}}$ assuming the luminosities (13). For each channel, the first row corresponds to the $e^{+} e^{-}$c.m. energy $\sqrt{s}=4.18 \mathrm{GeV}$ and the second one to $4.95 \mathrm{GeV}$. In the case of $a \neq b$, the cross section value $\sigma_{a b}=\left(\sigma_{a \bar{b}}+\sigma_{b \bar{a}}\right)$. For the modes where leptons are involved, the cross section is the sum of the respective cross sections for $\ell=e$ and $\mu$; in particular, $\sigma_{\ell \ell^{\prime}}$ is the sum of the diagonal and nondiagonal channels.

\begin{tabular}{|c|c|c|c|c|c|c|c|c|}
\hline$\tau^{-} \rightarrow$ & $\tau^{+} \rightarrow$ & $\sigma_{a b}(\mathrm{pb})$ & $w_{a \bar{b}}$ & $\sqrt{\left\langle\left(\mathcal{O}_{R}^{a \bar{b}}\right)^{2}\right\rangle_{0}}$ & $\delta \operatorname{Re} d_{\tau}\left(\times 10^{-17} e \mathrm{~cm}\right)$ & $\omega_{a \bar{b}}$ & $\sqrt{\left\langle\left(\mathcal{O}_{I}^{\prime a \bar{b}}\right)^{2}\right\rangle_{0}}$ & $\delta \operatorname{Im} d_{\tau}\left(\times 10^{-17} e \mathrm{~cm}\right)$ \\
\hline \multirow[t]{2}{*}{$\pi^{-} \nu$} & $\pi^{+} \bar{\nu}$ & 35.97 & 0.047 & 0.218 & 2.11 & 0.138 & 0.372 & 1.22 \\
\hline & & 31.26 & 0.085 & 0.292 & 2.45 & 0.235 & 0.485 & 1.47 \\
\hline \multirow[t]{2}{*}{$\pi^{-} \pi^{0} \nu$} & $\pi^{+} \pi^{0} \bar{\nu}$ & 170.13 & 0.049 & 0.221 & 0.94 & 0.142 & 0.377 & 0.55 \\
\hline & & 146.72 & 0.090 & 0.300 & 1.10 & 0.248 & 0.498 & 0.66 \\
\hline \multirow{2}{*}{$\pi^{-} \pi^{-} \pi^{+} \nu$} & $\pi^{+} \pi^{+} \pi^{-} \bar{\nu}$ & 19.51 & 0.050 & 0.224 & 2.76 & 0.145 & 0.381 & 1.62 \\
\hline & & 16.88 & 0.093 & 0.304 & 3.17 & 0.258 & 0.508 & 1.91 \\
\hline \multirow[t]{2}{*}{$\pi^{-} \nu$} & $\pi^{+} \pi^{0} \bar{\nu}$ & 156.22 & 0.048 & 0.220 & 1.00 & 0.141 & 0.375 & 0.58 \\
\hline & & 135.00 & 0.088 & 0.297 & 1.16 & 0.243 & 0.492 & 0.69 \\
\hline \multirow[t]{2}{*}{$\pi^{-} \nu$} & $\pi^{+} \pi^{+} \pi^{-} \bar{\nu}$ & 52.83 & 0.049 & 0.222 & 1.70 & 0.143 & 0.378 & 0.99 \\
\hline & & 45.59 & 0.090 & 0.300 & 1.96 & 0.250 & 0.499 & 1.18 \\
\hline \multirow[t]{2}{*}{$\pi^{-} \pi^{0} \nu$} & $\pi^{+} \pi^{+} \pi^{-} \bar{\nu}$ & 115.18 & 0.050 & 0.223 & 1.13 & 0.144 & 0.380 & 0.67 \\
\hline & & 99.41 & 0.092 & 0.303 & 1.32 & 0.254 & 0.504 & 0.79 \\
\hline \multirow[t]{2}{*}{$\ell^{-} \nu \bar{\nu}$} & $\ell^{\prime+} \bar{\nu} \nu$ & 372.47 & 0.002 & 0.045 & 3.18 & 0.028 & 0.166 & 0.84 \\
\hline & & 323.30 & 0.004 & 0.059 & 3.27 & 0.045 & 0.211 & 1.04 \\
\hline \multirow[t]{2}{*}{$\ell^{-} \nu \bar{\nu}$} & $\pi^{+} \bar{\nu}$ & 231.15 & 0.010 & 0.097 & 1.74 & 0.078 & 0.278 & 0.64 \\
\hline & & 200.54 & 0.017 & 0.129 & 2.14 & 0.127 & 0.356 & 0.79 \\
\hline \multirow[t]{2}{*}{$\ell^{-} \nu \bar{\nu}$} & $\pi^{+} \pi^{0} \bar{\nu}$ & 503.25 & 0.010 & 0.100 & 1.21 & 0.078 & 0.279 & 0.43 \\
\hline & & 434.88 & 0.017 & 0.131 & 1.47 & 0.129 & 0.359 & 0.53 \\
\hline \multirow[t]{2}{*}{$\ell^{-} \nu \bar{\nu}$} & $\pi^{+} \pi^{+} \pi^{-} \bar{\nu}$ & 170.28 & 0.010 & 0.100 & 2.08 & 0.079 & 0.281 & 0.74 \\
\hline & & 147.00 & 0.018 & 0.133 & 2.43 & 0.132 & 0.364 & 0.91 \\
\hline
\end{tabular}

observables and the square roots of their variances for the $\tau^{\mp}$ decays listed above to one, two, and/or three measured particles. The 1 s.d. statistical sensitivities to $\operatorname{Re} d_{\tau}(s)$ and $\operatorname{Im} d_{\tau}(s)$ given in this table were computed in analogy to (17) with the listed cross sections and the assumed integrated luminosities (13). The numbers show that, as expected, the optimal observables $\mathcal{O}_{R}$ and $\mathcal{O}_{I}^{\prime}$ are significantly more sensitive to the $\tau$ EDM than the observables $T_{33}$ and $Q^{\prime}{ }_{33}$. Taking into account the full kinematic information on the hadronic system in the $\tau \rightarrow 2 \pi \nu_{\tau}$ and $\tau \rightarrow 3 \pi \nu_{\tau}$ decays, as we do here, results in maximal $\tau$-spin analyzing power [12,13], as in the decay $\tau \rightarrow \pi \nu_{\tau}$; i.e., the values of the coefficients $w_{a \bar{b}}(s)$ and $\omega_{a \bar{b}}(s)$ are equal for the respective channels. However, this holds only if no cuts are applied. The application of cuts to the various $n$-meson final states leads to slight distortions as the numbers in Table III show.
Assuming uncorrelated errors, we can add in quadrature the statistical errors of $\operatorname{Re} d_{\tau}$ and $\operatorname{Im} d_{\tau}$ listed in Tables I, II, and III for the various channels,

$$
\delta \operatorname{Re} d_{\tau}=\left(\sum_{a b} \frac{1}{\left(\delta \operatorname{Re} d_{\tau}\right)_{a b}^{2}}\right)^{-1 / 2},
$$

and analogously for $\delta \operatorname{Im} d_{\tau}$. The results of these quadratures are given in Table IV. The sensitivity to $\operatorname{Re} d_{\tau}$ is improved by a factor of about 6 with the optimal observable $\mathcal{O}_{R}$ as compared to using $T_{33}$, while the improvement of the sensitivity to $\operatorname{Im} d_{\tau}$ by using $\mathcal{O}_{I}^{\prime}$ is slightly smaller.

The optimal observables involve the momenta of charged leptons and/or charged and neutral pions from $\tau^{ \pm}$decay in the respective $\tau^{ \pm}$rest frame. If both $\tau^{+}$and $\tau^{-}$ decay semihadronically or if one of the $\tau$ leptons decays semihadronically and the other one to either $e$ or $\mu$, the

TABLE IV. Ideal 1 s.d. statistical errors on $\operatorname{Re} d_{\tau}$ and $\operatorname{Im} d_{\tau}$ that result from adding the respective uncertainties given in Tables I, II, and III in quadrature. The first row corresponds to the $e^{+} e^{-}$c.m. energy $\sqrt{s}=4.18 \mathrm{GeV}$ and the second one to $4.95 \mathrm{GeV}$.

\begin{tabular}{lccccc}
\hline \hline$\delta \operatorname{Re} d_{\tau}(e \mathrm{~cm})$ & \multicolumn{5}{c}{$\delta \operatorname{Im} d_{\tau}(e \mathrm{~cm})$} \\
\hline$\left.T_{33}\right\rangle_{a b}$ & $\left\langle\hat{T}_{33}\right\rangle_{a b}$ & $\left\langle\mathcal{O}_{R}^{a b}\right\rangle$ & $\left\langle Q_{33}^{\prime}\right\rangle_{a b}$ & $\left\langle\hat{Q}^{\prime}{ }_{33}\right\rangle_{a b}$ & $\left\langle\mathcal{O}_{I}^{\prime a b}\right\rangle$ \\
$2.6 \times 10^{-17}$ & $3.3 \times 10^{-17}$ & $4.5 \times 10^{-18}$ & $1.0 \times 10^{-17}$ & $1.6 \times 10^{-17}$ & $2.1 \times 10^{-18}$ \\
$3.0 \times 10^{-17}$ & $3.7 \times 10^{-17}$ & $5.3 \times 10^{-18}$ & $1.0 \times 10^{-17}$ & $1.5 \times 10^{-17}$ & $2.6 \times 10^{-18}$ \\
\hline \hline
\end{tabular}


TABLE V. Ideal 1 s.d. statistical errors on $\operatorname{Re} d_{\tau}$ and $\operatorname{Im} d_{\tau}$ that result from adding in quadrature the respective uncertainties given in Table III attainable with the optimal observables $\mathcal{O}_{R}^{a b}$ and $\mathcal{O}_{I}^{\prime a b}$ in the semihadronic decays $(h h)$ and in the semihadronic and semihadronic-leptonic $(h h+h \ell)$ decays of $\tau^{+} \tau^{-}$. The respective first row corresponds to the $e^{+} e^{-}$c.m. energy $\sqrt{s}=$ $4.18 \mathrm{GeV}$ and the second one to $4.95 \mathrm{GeV}$.

\begin{tabular}{lcc}
\hline \hline & $\delta \operatorname{Re} d_{\tau}(e \mathrm{~cm})$ & $\delta \operatorname{Im} d_{\tau}(e \mathrm{~cm})$ \\
\hline$h h:$ & $5.3 \times 10^{-18}$ & $3.1 \times 10^{-18}$ \\
& $6.1 \times 10^{-18}$ & $3.7 \times 10^{-18}$ \\
$h h+h \ell:$ & $4.5 \times 10^{-18}$ & $2.1 \times 10^{-18}$ \\
& $5.3 \times 10^{-18}$ & $2.7 \times 10^{-18}$ \\
\hline \hline
\end{tabular}

rest-frame momenta of these final-state particles can be reconstructed from their measured momenta in the laboratory frame and kinematic constraints, using the method of [14]; see the corresponding discussion in Sec. 5 of [2]. If both $\tau$ leptons decay leptonically, the determination of their momenta is not possible in an unambiguous way.

Discarding the results for the $\ell \ell^{\prime}$ channels and adding in quadrature, the statistical errors of $\operatorname{Re} d_{\tau}$ and $\operatorname{Im} d_{\tau}$ listed in Table III where (i) both $\tau$ 's decay semihadronically and (ii) where the semihadronic-leptonic decays of $\tau^{+} \tau^{-}$are added to the purely semihadronic events we obtain the 1 s.d. errors given in Table V. Comparing the numbers in this table with those in Table IV shows that restriction to these $\tau^{+} \tau^{-}$decays does not significantly decrease the sensitivity to $\operatorname{Re} d_{\tau}$ and $\operatorname{Im} d_{\tau}$.

\section{CONCLUSIONS}

We found that the prospects are promising of improving the sensitivity in the search for a nonzero $\tau$ EDM at the BEPC-II collider compared to existing upper bounds on
$\operatorname{Re} d_{\tau}$ and $\operatorname{Im} d_{\tau}$. We considered $\tau^{+} \tau^{-}$production and decay at the $e^{+} e^{-}$c.m. energies $\sqrt{s}=4.18$ and $4.95 \mathrm{GeV}$. At these energies, the BESIII experiment has already recorded a large number of $\tau$-pair events. With realistic phase-space cuts on the measured final-state particles and realistic assumptions on the eventually attainable event numbers, we found that by taking into account purely semihadronic and semihadronic-leptonic $\tau^{+} \tau^{-}$decays one can obtain with the optimal $C P$-odd observables defined above the 1 s.d. sensitivities $\delta \operatorname{Re} d_{\tau}=4.5 \times 10^{-18} e \mathrm{~cm} \quad\left(5.3 \times 10^{-18} e \mathrm{~cm}\right)$ and $\delta \operatorname{Im} d_{\tau}=2.1 \times 10^{-18} e \mathrm{~cm}\left(2.7 \times 10^{-18} e \mathrm{~cm}\right)$ at $\sqrt{s}=$ $4.18 \mathrm{GeV}(4.95 \mathrm{GeV})$. Assuming that the EDM form factor $d_{\tau}(s)$ varies only moderately with the c.m. energy in the interval $4.1 \mathrm{GeV}<\sqrt{s}<5 \mathrm{GeV}$, we can, for instance for the $h h+h \ell$ case, combine the sensitivities calculated for the two values of $\sqrt{s}$ listed in Table $\mathrm{V}$. We then get the 2 s.d. sensitivities

$\delta \operatorname{Re} d_{\tau}=6.9 \times 10^{-18} e \mathrm{~cm}, \quad \delta \operatorname{Im} d_{\tau}=3.3 \times 10^{-18} e \mathrm{~cm}$,

obtainable with the BESIII experiment with the integrated luminosities (13). Since 2 s.d. corresponds roughly to a 95\% confidence-level interval, we can compare these numbers with the best limits (1) available to date. This shows that improvement of the precision of $\operatorname{Re} d_{\tau}$ and $\operatorname{Im} d_{\tau}$ by a factor of around 5 should be feasible by the BESIII experiment.

\section{ACKNOWLEDGMENTS}

The authors thank Frank Nerling for discussions and correspondence. The work of L.C. was supported by the Deutsche Forschungsgemeinschaft under Grant No. 396021762-TRR 257.
[1] K. Inami et al. (Belle Collaboration), Search for the electric dipole moment of the $\tau$ lepton, Phys. Lett. B 551, 16 (2003).

[2] W. Bernreuther, L. Chen, and O. Nachtmann, Electric dipole moment of the tau lepton revisited, Phys. Rev. D 103, 096011 (2021).

[3] T. Abe et al. (Belle-II Collaboration), Belle II technical design report, arXiv:1011.0352.

[4] E. Kou et al. (Belle-II Collaboration), The Belle II physics book, Prog. Theor. Exp. Phys. 2019, 123 C01 (2019); 2020, 029201(E) (2020).

[5] X. Chen and Y. Wu, Search for the Electric Dipole Moment and anomalous magnetic moment of the tau lepton at tau factories, J. High Energy Phys. 10 (2019) 089.
[6] M. Ablikim et al. (BESIII Collaboration), Future physics programme of BES III, Chin. Phys. C 44, 040001 (2020).

[7] W. Bernreuther and O. Nachtmann, $C P$ Violating Correlations in Electron Positron Annihilation Into $\tau$ Leptons, Phys. Rev. Lett. 63, 2787 (1989); 64, 1072(E) (1990).

[8] W. Bernreuther, O. Nachtmann, and P. Overmann, The $C P$ violating electric and weak dipole moments of the $\tau$ lepton from threshold to 500-GeV, Phys. Rev. D 48, 78 (1993).

[9] D. Atwood and A. Soni, Analysis for magnetic moment and electric dipole moment form-factors of the top quark via $e^{+} e^{-} \rightarrow t \bar{t}$, Phys. Rev. D 45, 2405 (1992).

[10] M. Davier, L. Duflot, F. Le Diberder, and A. Rouge, The optimal method for the measurement of tau polarization, Phys. Lett. B 306, 411 (1993). 
[11] M. Diehl and O. Nachtmann, Optimal observables for the measurement of three gauge boson couplings in $e^{+} e^{-} \rightarrow W^{+} W^{-}$, Z. Phys. C 62, 397 (1994).

[12] A. Rougé, Polarization observables in the $3 \pi \nu$ decay mode of the $\tau$, Z. Phys. C 48, 75 (1990).
[13] J. H. Kühn, $\tau$ polarimetry with multimeson states, Phys. Rev. D 52, 3128 (1995).

[14] J. H. Kühn, Tau kinematics from impact parameters, Phys. Lett. B 313, 458 (1993). 